\title{
Non-neoplastic asbestos-related respiratory diseases: what relationship with other lung diseases?
}

\author{
Sartorelli $\mathbf{P}^{1 *}$, Bianchi $F^{2}$ and Paolucci $\mathbf{P}^{1}$ \\ ${ }^{1}$ Department of Medical Biotechnology, Unit of Occupational Medicine, Azienda Ospedaliera Universitaria Senese, University of Siena, Siena, Italy \\ ${ }^{2}$ Department of Medical, Surgical and Neuroscience, Unit of Respiratory Diseases and Lung Transplant, Azienda Ospedaliera Universitaria Senese, University of \\ Siena, Siena, Italy
}

\begin{abstract}
Non-neoplastic asbestos-related respiratory diseases in practice are represented by asbestosis and pleural plaques. Clinical features and architectural tissue abnormalities of asbestosis do not differ from those of other causes of interstitial fibrosis which allows a confident diagnosis without a history of significant exposure to asbestos. But the relationship between non-neoplastic asbestos-related diseases and other lung diseases is not only represented by the need for differential diagnosis. developing lung diseases not classified as Asbestos-Related Diseases.

The article reviews the existing relationship between non-neoplastic asbestos-related respiratory diseases and interstitial lung diseases, lung function and risk of
\end{abstract} pleuropulmonary neoplastic diseases.

\section{Background}

The epidemic of Asbestos-Related Diseases (ARDs) is spread all over the world. If in USA illnesses due to occupational asbestos exposure are decreasing, in other Countries, such as Italy and Japan they are still increasing. In Italy, asbestos production reached a peak in the period between 1976-1980, but remained steady over 100,000 tons/ year until 1987. These temporal patterns delayed the peak in asbestos consumption in Italy compared to other European countries and in the United States [1]. Moreover, the use of asbestos continues to increase in Asia. Most ARDs are pleuropulmonary. In practice, non-neoplastic ARDs are represented by asbestosis and Pleural Plaques (PPs). Clinical features and architectural tissue abnormalities of asbestosis do not differ from those of other causes of interstitial fibrosis which does not allow a confident diagnosis without a history of significant exposure to asbestos [2,3]. Epidemiologic data on occupational interstitial lung diseases in general is limited by non-standardized diagnostic criteria, varied physician awareness and training, limitations inherent to the various data sources and the long latency period [4]. This leads to underreport occupational lung diseases. But the relationship between non-neoplastic ARDs and other lung diseases is not only represented by the need for differential diagnosis. A diagnosis of non-malignant ARDs does imply a lifelong elevated risk to asbestos-related cancer, while asbestos exposure itself could increase the risk of developing lung diseases not classified as ARDs [5].

This article reviews the existing relationship between nonneoplastic ARDs and interstitial lung diseases, lung function and risk of pleuropulmonary neoplastic diseases.

\section{Asbestosis and other interstitial lung diseases}

Asbestosis is an interstitial lung fibrosis caused by the inhalation of all types of asbestos fibers. A histological diagnosis of asbestosis requires the identification of diffuse interstitial fibrosis plus the presence of either 2 or more asbestos bodies in tissue with a section area of $1 \mathrm{~cm}^{2}$ or a count of uncoated asbestos fibres that falls in the range recorded for asbestosis by the same laboratory [3]. Being the fibrosis dose-dependent, asbestosis can be induced by cumulative asbestos exposure amounting to an estimated 25 fibres/ml-yrs lower levels can also cause disease in some workers $[4,6]$. It is well known that the presence of asbestosis increases the risk of lung cancer. In contrast to the asbestosis seen in the past, today most cases of asbestosis are asymptomatic disease identified on radiologic investigation or by histologic assessment of lung parenchyma remote from a resected lung cancer [7]. Usually the diagnosis of asbestosis is based on clinical findings and radiology in the absence of histology. The main differential diagnosis is with idiopathic lung fibrosis, in particular Usual Interstitial Pneumonia (UIP). Pathologically asbestosis is characterized by the fibrosis of alveolar walls adjacent to the respiratory bronchioles, which extend to involve the surrounding lung in the centrifugal direction. In contrast UIP begins at the periphery of the secondary pulmonary lobule and progresses in the centripetal direction. These anatomical differences of lung fibrosis could be appreciated by High-Resolution Computed Tomography (HRCT) images to some extent, not without difficulty [8]. HRCT features include increased intralobular septal markings, subpleural curvilinear lines, parenchymal bands and small cysts $[7,9]$. The clinic can help since progression of asbestosis, as in other pneumoconiosis, is slower compared to idiopathic interstitial fibrosis. To complicate matters it is suspected that asbestos influences the risk of developing UIP, but the findings are inconclusive [4]. A significant linear relationship was demonstrated in the UK between mortality due to interstitial lung fibrosis and historic asbestos imports

Correspondence to: Pietro Sartorelli, Department of Medical Biotechnology, Unit of Occupational Medicine, Azienda Ospedaliera Universitaria Senese, University of Siena, Siena, Italy, E-mail. pietro.sartorelli@unisi.it

Key words: asbestos, asbestosis, lung diseases, pleural diseases, occupational exposure, smoking, respiratory function test

Received: June 14, 2017; Accepted: June 26, 2017; Published: June 28, 2017 
[10]. The strength of this association was similar to that seen in mesothelioma. This finding could be explained in part by diagnostic difficulties in separating asbestosis from interstitial lung fibrosis.

To determine whether UIP pattern fibrosis is seen in asbestosis Attanoos et al. studied the occurrence of UIP pattern fibrosis in four asbestos cohorts (17-year period, $>25000$ persons) systematically referred following post-mortem [11]. Out of 233 subjects 210 had degrees of interstitial fibrosis with a Nonspecific Interstitial Pneumonia (NSIP) pattern and 3 showed UIP pattern fibrosis. A Poisson distribution indicated that the observed cases of UIP in this workforce could be wholly accounted for by the prevalence of interstitial lung fibrosis in the general population. Authors concluded that UIP pattern fibrosis is rarely observed in asbestos-exposed subjects and shows no dose-response correlation with asbestos fibres on mineral analysis. Thus, UIP pattern fibrosis should not be regarded as genuine asbestosis irrespective of the status of asbestos biomarkers. However other Authors in the same year and the same Journal published different results [12]. They retrospectively examined 1718 cases who underwent lobectomy for the resection of pleuropulmonary tumours. Presence of malignant pleural mesothelioma and presence of PPs and asbestos bodies in the histological specimen were considered as markers for asbestos exposure. A group of 183 cases of asbestos-exposed workers was identified. Multivariate analysis showed that asbestos exposure (especially positivity for asbestos bodies that does not meet the current criteria for asbestosis) increases the risk of histological UIP $(\mathrm{p}<0.0001)$. The 183-asbestos exposed group showed a significantly higher incidence of histological UIP (31\%) in addition to higher incidence rates in males, being elderly and being a smoker than the non-exposed group. The same Authors in a previous article had reported that patients undergoing lobectomy for lung tumours who had PPs showed a significantly higher incidence of histological UIP [12].

In a Japanese study Computed Tomography (CT) images and pathology specimens of 33 workers with clinical diagnoses of asbestosis were collected [8]. 15 cases were diagnosed as asbestosis and 18 cases with various lung fibroses other than asbestosis. On CT only the score of the subpleural curvilinear lines was significantly higher in asbestosis. The asbestos body count positively correlated with CT likelihood of asbestosis, and with the confidence level of pathological diagnosis. The Authors concluded that various patterns of pulmonary fibrosis occurred in asbestos exposed workers and the fibre burden in lungs paralleled confident CT and pathological diagnosis of asbestosis.

Asbestos exposure may be associated with fibrosis of the walls of the respiratory bronchioles and alveolar ducts [7]. These lesions do not represent asbestosis. The asbestos Committee of the College of American Pathologists and Pulmonary Pathology Society proposed the term asbestos airways disease for bronchiolar wall fibrosis associated with asbestos bodies [7]. This respiratory bronchiolitis is similar to those observed from exposure to cigarette smoke and mineral dust. At the initial stage, it may be very difficult to diagnose asbestosis in cigarette smokers. Bledsoe et al. evaluate the correlation of radiographically detected pulmonary fibrosis with fibrosis established histopathologically as attributable to asbestos, in a cohort referred for diagnosis of an asbestos-related malignancy [13]. Criteria used for the diagnosis of smoking-associated interstitial fibrosis was a history of heavy smoking ( $>20$ pack-years), interstitial fibrosis of the pattern seen in smoking-associated fibrosis and presence of emphysema or respiratory bronchiolitis. Of 24 cases judged to have asbestosis radiographically 6 showed asbestosis histopathologically. The remaining 18 cases (mean smoking history of 53 packs-years) showed interstitial fibrosis that was judged to be most consistent with smoking-associated pulmonary fibrosis. They conclude that the clinical diagnosis of mild asbestosis cannot be reliably distinguished from interstitial fibrosis in heavy smokers. Asbestos bodies and fibres in bronchoalveolar lavage fluid and in lung specimens are of great assistance in the differential diagnosis $[6,14]$.

\section{Asbestos, non-neoplastic asbestos-related respiratory diseases and lung function}

The possibility that asbestos exposure, in the absence of clinically diagnosed ARDs, may be associated with lung function impairment is discussed. A systematic review and meta-analysis demonstrated a statistically significant reduction in Vital Capacity (VC), Forced Expiratory Volume in $1 \mathrm{~s}$ (FEV1) and FEV1/VC, even in those workers without radiological changes. Even in the absence of radiological evidence of parenchymal or pleural diseases there was a trend for functional impairment. Authors concluded that asbestos exposure is related to restrictive and obstructive lung function impairment [15]. Another study described lung function profiles among patients with ARDs [16]. Significant differences in lung function in individuals with asbestos-related diseases compared to currently healthy individuals with a history of previous exposure to asbestos were found. Since occupational exposure to asbestos is strongly related to an increased decline in lung function, regular monitoring of lung function among asbestos exposed populations represents a simple tool to facilitate earlier interventions. Wang et al. studied the adverse effects of exposure to asbestos and smoking on pulmonary function analysis on 468 asbestos exposed workers and 282 controls [17]. Multivariate regression showed that exposure to asbestos was more strongly associated with decreased Forced Vital Capacity (FVC) and CO Diffusing Capacity (DLCO), and asbestosis more strongly associated with decreased FVC, while smoking was a major contributing factor to reduced FEV1/FVC. This analysis suggested that asbestos and smoking might play independent roles, in which asbestos caused mainly a restrictive impairment, and smoking was a major causal factor for airway obstruction in the workers who were intensively exposed to asbestos.

The decrease of lung function should be influenced by the progression of ARDs even after the cessation of exposure. Actually, both radiographic and functional pulmonary deterioration may occur long after asbestos exposure [3]. The changes in pulmonary function tests (FEV1, FVC, total lung capacity TLC, DLCO) and CT imaging of the thorax over a 15-year period after cessation of exposure to asbestos were described in a cohort of Israeli power plant workers [18]. Comparison of the initial and follow up examination findings revealed a significant increase in calcification of the PPs and a deterioration in pulmonary function tests results. This progression of ARDs in workers formerly exposed to asbestos who had not been exposed to it for over a decade recommends the continued monitoring of individuals exposed to asbestos after the cessation of exposure.

\section{Pleural plaques, lung function and risk of malignant asbestos-related diseases}

Several studies investigated the possible relationship between PPs and lung function, but the results are inconclusive. In various articles in literature an association between PPs and a slight degree of lung function impairment without clinical relevance was observed. However, most studies have demonstrated no significant association between PPs and abnormal pulmonary function tests [19]. Spanish guidelines for the diagnosis and management of asbestos-related pleural and 
pulmonary disease consider that PPs in general do not cause a decrease in pulmonary function and when it appears, it would be due to other concomitant causes, such as the onset of interstitial fibrosis or smoking [20]. On the other hand, a systematic review demonstrated that the presence of PPs is associated with a small, but statistically significant mean difference in FVC and FEV1 in comparison to asbestos exposed individuals without PPs or other abnormalities [21].

Another systematic review evaluated whether there is an association between PPs and lung function and if results were dependent on the method used to identify plaques [22]. 6 HRCT and 6 X-ray high quality studies were identified. Half of them reported small but statistically significant mean lung function decrements associated with plaques. None of the differences were clinically significant. X-ray results were more variable than HRCT results. PPs were not associated with changes in lung function over time in longitudinal studies. The weight of evidence indicated that PPs do not impact lung function and the observed associations were most likely due to unidentified abnormalities or other factors.

In another study, occupational asbestos exposure in a cohort of heavy smokers was associated with a significant restrictive decline in pulmonary function, with longer duration of exposure associated with greater decline. The presence of isolated PPs was also associated with reduced lung function [23].

A population of 2743 asbestos exposed workers with no interstitial abnormalities on the thorax HRCT was studied [24]. All functional parameters studied were within normal limits for subjects who presented isolated PPs and for those who presented no pleuropulmonary abnormalities, but PPs were associated with a significant decrease in TLC. No significant relationship was observed between PPs and other functional parameters. The results showed a relationship between PPs and a trend towards a restrictive pattern, although this was unlikely to be of real clinical relevance for the majority of subjects.

Despite an initial case report that raised concern there is broad agreement that PPs are not premalignant, even if Pairon et al. in 17 cases of pleural mesothelioma observed a statistically significant association between mesothelioma and PPs [25-27].

A review summarized the literature on the relationship between PPs and non-malignant and malignant disease in cohorts exposed to asbestos and other fibres [26]. In the absence of any other pleural disease the presence of PPs did not result in respiratory symptoms or clinically significant impacts on lung function. For certain types of asbestos, the development of PPs was statistically correlated with malignant disease, but the evidence was consistent with the hypothesis that PPs without other pleural disease are a marker of exposure rather than an independent risk factor. PPs have also developed in cohorts exposed to other fibres that have not proven to be carcinogenic. The conclusion was that risk analyses should be based on the avoidance of known adverse conditions rather than PPs per se.

A Japanese study found the existence of a relationship between the extent of PPs and pulmonary asbestos body concentration of 207 lung cancer patients [28]. Thus, the PPs extent was considered useful as a proxy for pulmonary asbestos body concentration for lung cancer compensation purposes.

\section{Conclusions}

Despite the number of publications on the subject in most cases the existence or not of a relationship between non-malignant ARDs and other lung diseases is still controversial. In particular interstitial lung fibrosis other than asbestosis have a very uncertain relationship with occupational exposure to asbestos. On the other hand, a number of studies demonstrated no significant association between PPs and lung function impairment, although there is still debate on this issue [19]. Data in the literature very often are conflicting and to date there are few certainties in the specific field. The results of health surveillance on the exposed workers populations probably will help to better understand the problem.

\section{References}

1. Corfiati M, Scarselli A, Binazzi A, Di Marzio D, Verardo M, et al. (2015) Epidemiological patterns of asbestos exposure and spatial clusters of incident cases of malignant mesothelioma from the Italian national registry. BMC Cancer 15: 286-299. [Crossref]

2. [No authors listed] (1997) Asbestos, asbestosis, and cancer: the Helsinki criteria for diagnosis and attribution. Scand J Work Environ Health 23: 311-316. [Crossref]

3. Wolff H, Vehmas T, Oksa P, Rantanen J, Vainio H (2015) Asbestos, asbestosis, and cancer, the Helsinki criteria for diagnosis and attribution 2014: recommendations. Scand J Work Environ Health 41: 5-15. [Crossref]

4. Glazer CS, Newman LS (2004) Occupational interstitial lung disease. Clin Chest Med 25: 467-478. [Crossref]

5. American Thoracic Society (2004) Official Statement: Diagnosis and initial management of non-malignant diseases related to asbestos. Am J Resp Crit Care Med 170: 691-715

6. Sartorelli P, Scancarello G, Romeo R, Marciano G, Rottoli P, et al. (2001) Asbestos exposure assessment by mineralogical analysis of bronchoalveolar lavage fluid. $J$ Occup Environ Med 43: 872-881. [Crossref]

7. Roggli VL, Gibbs AR, Attanoos R, Churg A, Popper H, et al. (2010) Pathology of asbestosis- An update of the diagnostic criteria: Report of the asbestosis committee of the College of American Pathologists and Pulmonary Pathology Society. Arch Pathol Lab Med 134: 462-480. [Crossref]

8. Arakawa I, Kishimoto T, Ashizawa K, Kato K, Okamoto K, et al. (2016) Asbestosis and other pulmonary fibrosis in asbestos-exposed workers: high-resolution $\mathrm{CT}$ features with pathological correlations. Eur Radiol 26: 1485-1492. [Crossref]

9. Akira M, Yamamoto S, Inoue Y, Sakatani M (2003) High-resolution CT of asbestosis and idiopathic pulmonary fibrosis. AJR Am J Roentgenol 181: 163-169. [Crossref]

10. Barber CM, Wiggans RE, Young C, Fishwick D (2016) UK asbestos imports and mortality due to idiopathic pulmonary fibrosis. Occup Med (Lond) 66: 106-111 [Crossref]

11. Attanoos RL, Alchami FS, Pooley FD, Gibbs AR (2016) Usual interstitial pneumonia in asbestos-exposed cohorts - concurrent idiopathic pulmonary fibrosis or atypical asbestosis? Histopathology 69: 492-498. [Crossref]

12. Kawabata Y, Shimizu Y, Hoshi E, Murai K, Kanauchi T, et al. (2016) Asbestos exposure increases the incidence of histologically confirmed usual interstitial pneumonia. Histopathology 68: 339-346. [Crossref]

13. Bledsoe JR, Christiani DC, Kradin RL (2014) Smoking-associated fibrosis and pulmonary asbestosis. Int J Chron Obstruct Pulmon Dis 10: 31-37. [Crossref]

14. Sartorelli P, Romeo R, Scancarello G, Montomoli L, Muzzupappa C, et al. (2007) Measurement of asbestos fibre concentrations in fluid of repeated bro-choalveolar lavages of exposed workers. Ann Occup Hyg 51: 495-500. [Crossref]

15. Wilken D, Velasco Garrido M, Manuwald U, Baur X (2011) Lung function in asbestosexposed workers, a systematic review and meta-analysis. J Occup Med Toxicol 6: 2136. [Crossref]

16. Park EK, Yates DH, Wilson D (2014) Lung Function Profiles among Individuals with Nonmalignant Asbestos-related Disorders. Saf Health Work 5: 234-237. [Crossref]

17. Wang X, Yano E, Wang Z, Wang M, Christiani DC (2006) Adverse effects of asbestos exposure and smoking on lung function. Am J Ind Med 49: 337-342. [Crossref]

18. Bar-Shai A, Tiran B, Topilsky M, Greif J, Fomin I, et al. (2012) Continued progression of asbestos-related respiratory disease after more than 15 years of non-exposure. Isr Med Assoc J 14: 560-565 [Crossref]

19. Prazakova S, Thomas PS, Sandrini A, Yates DH (2014) Asbestos and the lung in the 21 st century: an update. Clin Respir J 8: 1-10. [Crossref]

20. Diego Roza C, Cruz Carmona MJ, Fernandez Alvarez R, Ferrer Sancho J, Marin Martínez B, et al. (2017) Recommendations for the diagnosis and management of asbestos-related pleural and pulmonary disease. Arch Bronconeumol. [Crossref] 
21. Kopylev L, Christensen KY, Brown JS, Cooper GS (2015) A systematic review of the association between pleural plaques and changes in lung function. Occup Environ Med 72: 606-614. [Crossref]

22. Kerper LE, Lynch HN, Zu K, Tao G, Utell MJ, et al. (2015) Systematic review of pleural plaques and lung function. Inhal Toxicol 27: 15-44. [Crossref]

23. Lopatin S, Tsay JC, Addrizzo-Harris D, Munger JS, Pass H, et al. (2016) Reduced lung function in smokers in a lung cancer screening cohort with asbestos exposure and pleural plaques. $J$ Ind Med 59: 178-185. [Crossref]

24. Clin B, Paris C, Ameille J, Brochard P, Conso F, et al. (2011) Do asbestos-related pleural plaques on HRCT scans cause restrictive impairment in the absence of pulmonary fibrosis? Thorax 66: 985-91. [Crossref]
25. Lewinsohn HC (1974) Early malignant changes in pleural plaques due to asbestos exposure: a case report. Br J Dis Chest 68: 121-127. [Crossref]

26. Maxim LD, Niebo R, Utell MJ (2015) Are pleural plaques an appropriate endpoint for risk analyses? Inhal Toxicol 27: 321-334. [Crossref]

27. Pairon JC, Laurent F, Rinaldo M, Clin B, Andujar P, et al. (2013) Pleural plaques and the risk of pleural mesothelioma. J Natl Cancer Inst 105: 293-301. [Crossref]

28. Yusa T, Hiroshima K, Sakai F, Kishimoto T, Ohnishi K, et al. (2015) Significant relationship between the extent of pleural plaques and pulmonary asbestos body concentration in lung cancer patients with occupational asbestos exposure. Am J Ind Med 58: 444-455. [Crossref]

Copyright: $@ 2017$ Sartorelli P. This is an open-access article distributed under the terms of the Creative Commons Attribution License, which permits unrestricted use, distribution, and reproduction in any medium, provided the original author and source are credited. 